\title{
Fabrication of nontoxic natural dye from sappan wood
}

\author{
Dong-Kyu Lee, Dong-Hoe Cho, Jin-Hui Lee and Hun Yong Shin \\ Department of Chemical Engineering, Seoul National University of Technology, Seoul 139-743, Korea \\ (Received 10 July $2007 \cdot$ accepted 11 December 2007)
}

\begin{abstract}
The use of natural dyes has attracted increasing worldwide attention as the carcinogenicity and environmental pollution problem of synthetic pigments are becomes a great concern. A nontoxic natural dye was fabricated from an extract from Caesalpinia Sappan (sappan wood). Oily natural dye for industrial application was made from extracted sappan wood by using a micro-emulsion method. The absorbance of the dye extracted from sappan wood by distillation was measured with an Ultraviolet/Visible spectrometer. The highest absorbance was obtained from the mordant containing $2 \mathrm{wt} \% \mathrm{Al}$. The emulsification conditions were controlled by changing the mixing ratios of TEOS (tetraethyl ortho-silicate), DC3225C (cyclomethicone and dimethicon copolyol) and extracted dye. A W/O (water in oil) type dye was obtained with a TEOS concentration ranging from 19.9 to $50.0 \mathrm{wt} \%$ when the DC3225C concentration was fixed to $10 \mathrm{wt} \%$. The W/O type emulsion was verified by optical microscopy. The fabricated dye was applied to colored paper. The applicability of the nontoxic natural dye was verified by analyzing the heavy metals and color fastness of the colored paper.
\end{abstract}

Key words: Natural Dye, Extraction, Mordant, W/O Type, Sappan Wood

\section{INTRODUCTION}

Pigments obtained from plants, animals and insects have been used as food colorings and dyes. However, the use of natural dye extracted from raw material is restricted due to the scarcity of the source as well as the complicated extraction process and dyeing procedure. Since the introduction of synthetic dyes by Perkin in 1856, many convenient and cheap synthetic pigments have appeared, and the use of natural dyes has decreased due to their poor price competitiveness [1]. Recently, as living standards have improved and the carcinogenicity and environmental pollution problem of synthetic pigments has become a great concern, the use of natural dyes has attracted increasing worldwide attention. Traditionally, a natural dye means a pigment obtained from organisms. It also includes natural dyes chemically modified for the stability, solubility and chromaticity of the pigment elements [2].

Traditional Korean natural dyes are obtained from flowers, leaves and the roots of plants [3]. The red color from sappan wood [4,5], yellow color from gardenia seeds [6], red color from safflower [7], purple color from gardenia, red color from madder and blue color from the indigo plant are typically used. Blue, red and yellow colors are essential for a competitive nature dye, and other colors can be made by mixing these three basic colors. A natural dye has a reverberating, subdued and deep impression of color. Furthermore, various brightnessess and color tones can appear as a result of mordants. However, the weak color fastness, complicated dyeing procedure and difficulty in long-term deposition are the weak points of natural dyes [8].

A natural dye should have an oily nature before it can be applied in industry such as colored paper. Oily natural dye can be made by using a micro-emulsion method that is used for the synthesis of mi-

${ }^{\dagger}$ To whom correspondence should be addressed.

E-mail: hyshin@snut.ac.kr cron-sized micelle particles.

By adding a surfactant, the micro-emulsion method utilizes a diffusion reactor by mixing the water and oil phases. The surfactants have both hydrophilic groups and hydrophobic groups. If the hydrophobic properties are stronger than the hydrophilic properties, the micelles formed will have an external oil layer and an internal water layer. This $\mathrm{W} / \mathrm{O}$ (water in oil) type of emulsion has been applied in various industries such as food, cosmetics, medicines, paints and petroleum [9].

In this study, the red color was extracted from sappan wood, which is a red pleochroism dye. By adding various mordants, the change in color was examined as a function of the mordant concentration. The extracted solutions were emulsified to the W/O type by the emulsifiers for the oily natural dye. Also, red colored paper was made by pasting the mixture of the emulsified extracted solution and acrylic resin. The applicability of the nontoxic natural dye was verified by analyzing the heavy metal and color fastness of the colored paper.

\section{EXPERIMENTAL SECTION}

\section{Material}

Sappan imported from china was used after washing. TEOS (tetraethyl orthosilicate 98\%, ACROS Co.) and DC3225C (cyclomethicone and dimethicon copolyol, DOW Coring $\mathrm{Co}$.) were used as emulsifier. Aluminum Potassium Sulfate $\left(\mathrm{AlK}\left(\mathrm{SO}_{4}\right)_{2} \cdot 12 \mathrm{H}_{2} \mathrm{O}\right)$, Ferrous Chloride $\left(\mathrm{FeCl}_{2} \cdot 4 \mathrm{H}_{2} \mathrm{O}\right)$, Copper(II) Acetate Monohydrate $(\mathrm{Cu}$ $\left.\left(\mathrm{CH}_{3} \mathrm{COO}\right)_{2} \cdot \mathrm{H}_{2} \mathrm{O}\right)$ and $\mathrm{Tin}(\mathrm{II})$ Chloride $\left.\left(\mathrm{SnCl}_{2} \cdot 2 \mathrm{H}_{2} \mathrm{O}\right)\right)$ from Junsei Chemical Co were used as mordants.

\section{Extraction of Dye}

Sappan wood contains brazilein as a pigment. Fig. 1 shows the chemical structure $[10,11]$. Twenty grams of sappan wood was washed with distilled water for 30 minutes and dried. Ten grams of dried sappan wood was poured in the flask, and the first extraction was obtained by heating with $500 \mathrm{ml}$ of distilled water for 60 minutes 


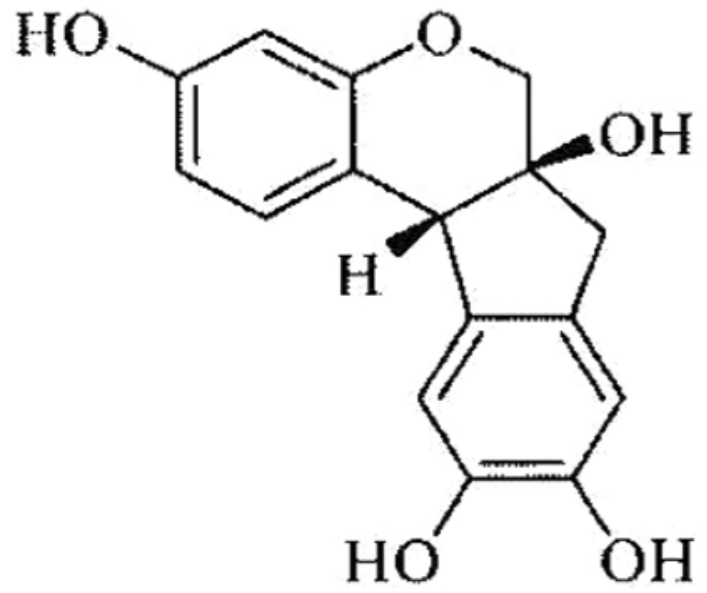

Fig. 1. The chemical structure of brazilein.

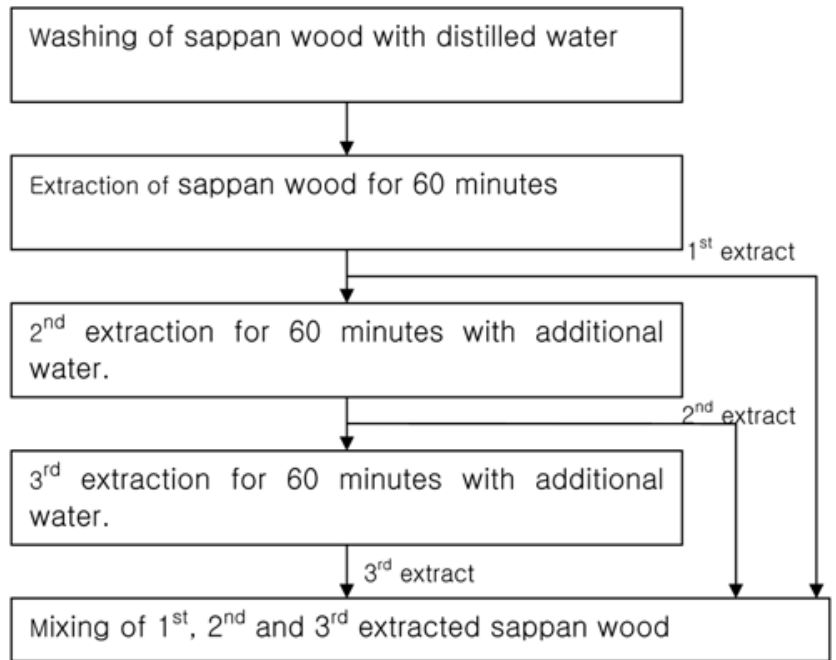

Fig. 2. Schematic diagram of the extraction procedure of sappan wood.

at $80^{\circ} \mathrm{C}$. The second extraction was obtained by heating the remaining sappan wood after mixing the first extraction and an additional $300 \mathrm{ml}$ of distilled water. The third extraction was obtained by heating with an additional $100 \mathrm{ml}$ of water for 60 minutes [12]. A mixture of the first, second and third exactions was used as the dye for emulsification. Fig. 2 shows a schematic diagram of the extraction procedure.

\section{Measurement of Absorbance}

The absorbance of the pigments from sappan wood was measured with an Ultraviolet/Visible Spectrometer (JASCO Co. JP/V560 ) according to the variation in mordants. Mordants such as Al, $\mathrm{Fe}, \mathrm{Cu}$ and $\mathrm{Sn}$ were added to the sappan wood dye, and the absorbance of the dye was measured. The optimum mordant with the maximum absorbance was selected by comparing the maximum absorption wavelength and absorbance according to the mordant. The optimum mordant and its concentration were examined by comparing the absorbance according to the mordant concentration of the selected mordant $[13,14]$.

\section{Emulsification}

Fig. 3 shows the W/O type micelle and Fig. 4 shows a sche-

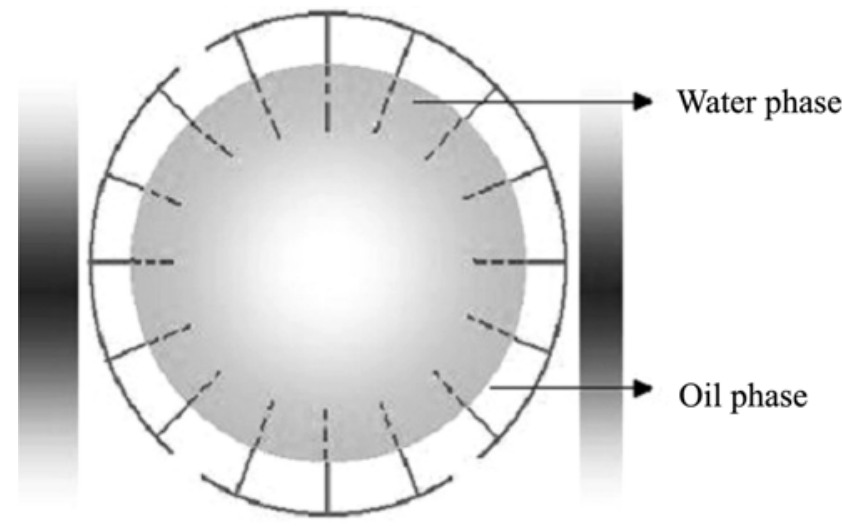

Fig. 3. Microsphere of W/O type emulsion.

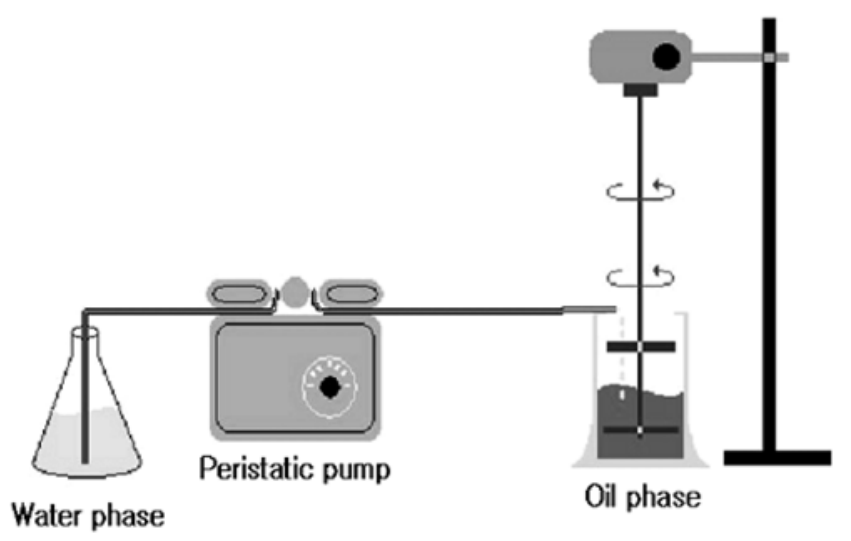

Fig. 4. Schematic diagram of the emulsion process.

matic diagram of the emulsification apparatus. A micro tube pump (PLKAKIKAI Co. LTD, Tokyo) was used to control of the flow rate. The extracted dye (solution 1) was mixed with a TEOS and DC3225C mixture (solution 2). Solution 1 was added to solution 2 at the rate of $1.9 \mathrm{ml} / \mathrm{min}$ and stirred at the rate of $750 \mathrm{rpm}$ for 30 minutes to complete the $\mathrm{W} / \mathrm{O}$ type emulsion.

The structure and shape of W/O type emulsion was examined by optical microscopy (Olympus. CK40-F200).

5. Particle Size and Heavy Metal Analysis

The physical characteristics of the W/O type emulsion particles were examined with a Lager Particle Analyzer (Otsuka Electronics. JP/PAT-III). The heavy metals in the synthesized W/O type pigment were analyzed by inductively coupled plasma (ICP, Perkin Elmer. 3300XL).

\section{Characteristics of Colored Paper}

A red ink for colored paper was made by pasting the mixture of the emulsified dye and acrylic resin which was supported by Micom Art Paper Co. Emulsified oily natural dye was mixed with $50 \mathrm{wt} \%$ of acrylic resin to paste on the paper. The synthesized ink was tested for its color fastness to sunlight by using the KS K 0700-1990 test method. The total solid after the vaporization and concentration of formaldehyde was measured by using the test method of the Korea Food and Drug Administration Notice No. 2000-60.

\section{RESULT AND DISCUSSION}

Korean J. Chem. Eng.(Vol. 25, No. 2) 


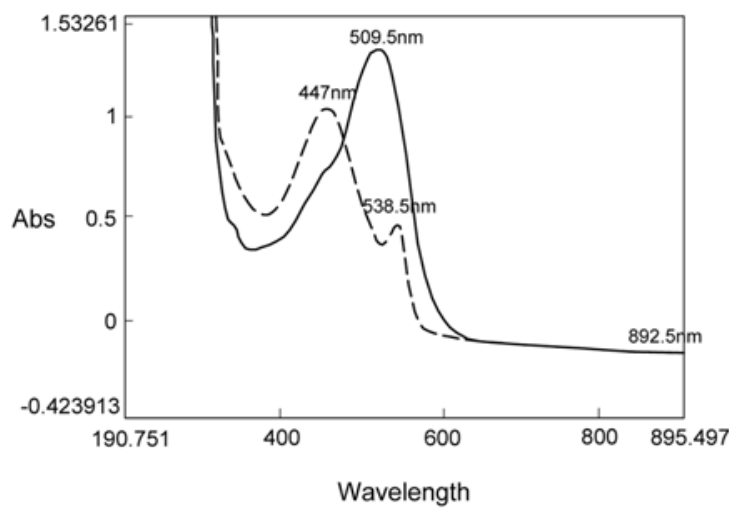

(a) $\mathrm{Al}$

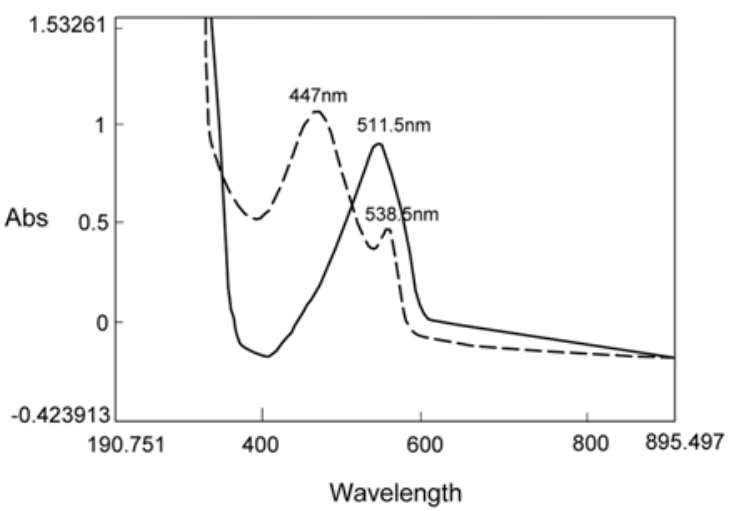

(c) $\mathrm{Sn}$

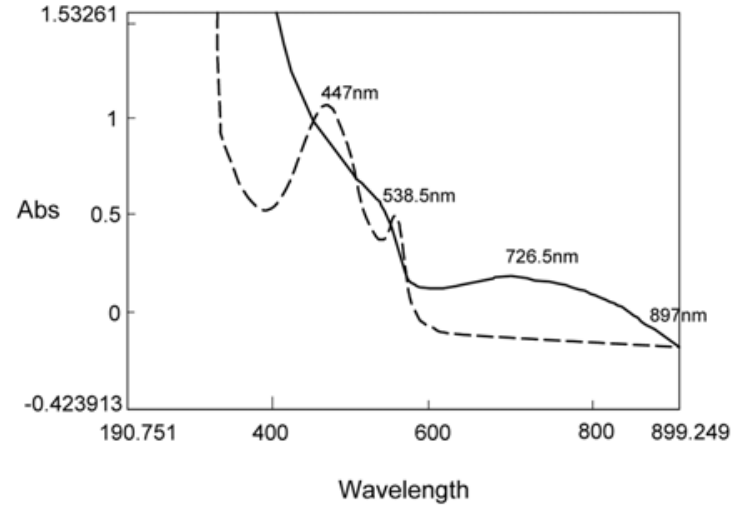

(b) $\mathrm{Fe}$

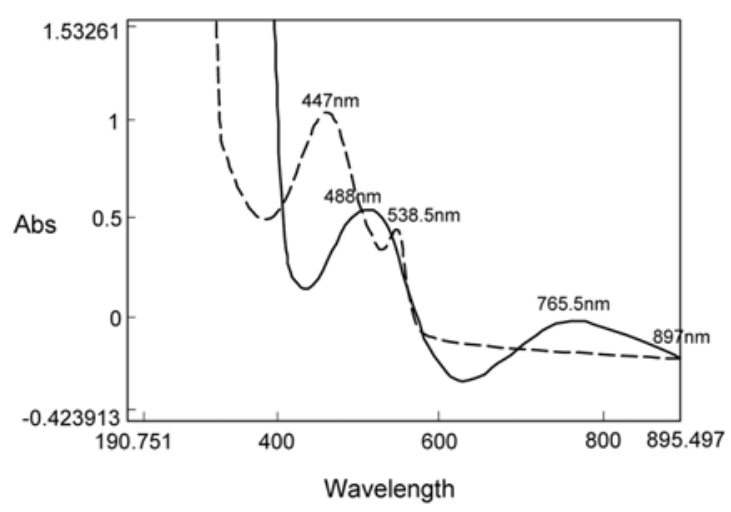

(d) $\mathrm{Cu}$

Fig. 5. Chemical shift of the wavelength by various mordant (solid line: addition of mordant, dashed line: no mordant).

\section{Absorption Wavelength and Absorbance by Addition of Mordant}

Fig. 5 shows the absorption wavelengths and absorbance according to the addition of $0.5 \mathrm{wt} \%$ mordant to the extracted sappan wood. The maximum absorption wavelengths were shifted according to the mordant. The maximum absorption wavelength of sappan wood by UV-spectrum was observed at 447 and $538 \mathrm{~nm}$. This indicates that sappan wood is a multicolored material containing yellow and red components. When a mordant of $\mathrm{Al}, \mathrm{Fe}, \mathrm{Sn}$ and $\mathrm{Cu}$ was used, the maximum absorption wavelength of red color was $509.5 \mathrm{~nm}$ (Fig. 5(a)), $726.5 \mathrm{~nm}$ (Fig. 5(b)), $511.5 \mathrm{~nm}$ (Fig. 5(c)) and $488 \mathrm{~nm}$ (Fig. 5(d)), respectively. In the case of the Fe mordant, the shift in the absorption wavelength to a higher wavelength was significant. A comparison of the maximum absorption wavelength showed the highest absorbance with the $\mathrm{Al}$ mordant; therefore, it was selected as the optimum mordant for a natural dye with sappan wood.

Fig. 6 shows the measured absorbance as a function of the $\mathrm{Al}$ mordant concentration. The absorbance increased according to the concentration of the $\mathrm{Al}$ mordant. However, the absorbance decreased above an $\mathrm{Al}$ mordant concentration of $2 \mathrm{wt} \%$. Although the concentration of pigment would increase with increasing $\mathrm{Al}$ mordant concentration, the concentration of pigment would be expected to decrease above an $\mathrm{Al}$ mordant concentration of $2 \mathrm{wt} \%$.

\section{Emulsification of Extracted Sappan Wood}

The extracted sappan wood was emulsified by changing the TEOS and DC3225C concentrations. The emulsification condition was

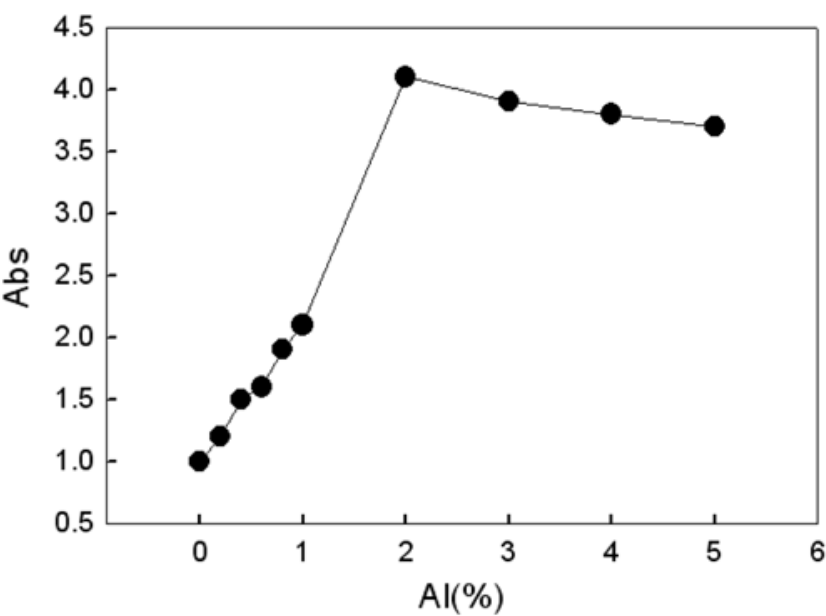

Fig. 6. Absorbance of dye according to aluminum mordant concentration (0-5 wt $\%$ of $\mathrm{Al}$ mordant+extracted dye).

dependent on the ratio of the water (extracted dye) and oil phases (TEOS and DC3225C mixture), and the possible region of emulsification was examined. In Fig. 7, one phase of emulsion was obtained at TEOS concentrations ranging from 19.9 to $50.0 \mathrm{wt} \%$ when the DC3225C concentration was fixed to $10 \mathrm{wt} \%$. Phase separation was observed below $19.9 \mathrm{wt} \%$ and above $50.0 \mathrm{wt} \%$ of TEOS. 


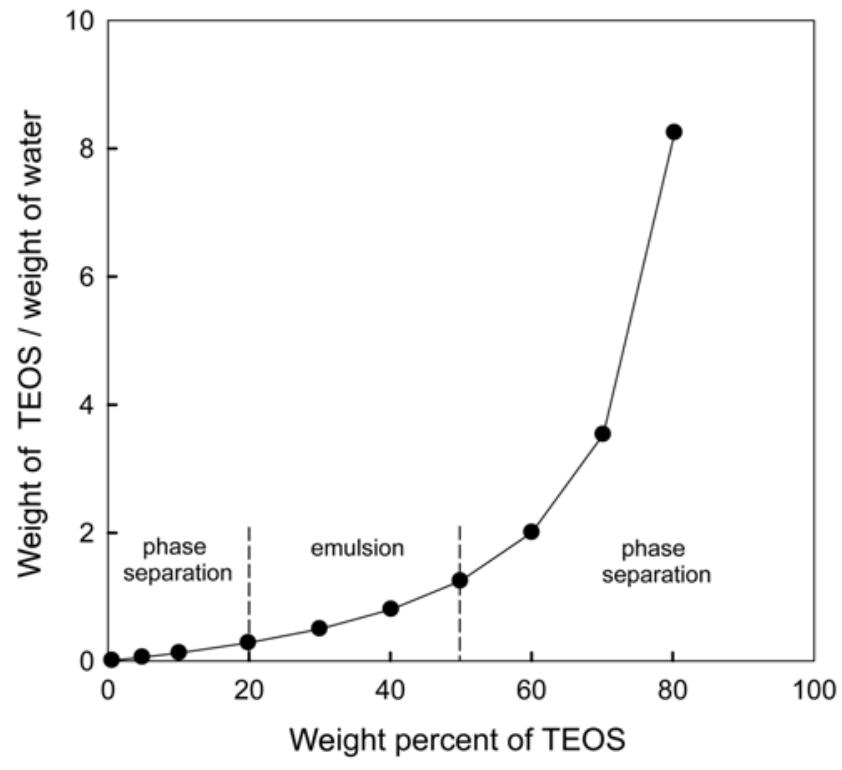

Fig. 7. Stability of water-in-oil type emulsion for various TEOS concentration at $25^{\circ} \mathrm{C}$ (injection rate of extracted dye: $1.9 \mathrm{ml} /$ min; stirring rate: $750 \mathrm{rpm}$; DC3225C: $10 \mathrm{wt} \%$ ).

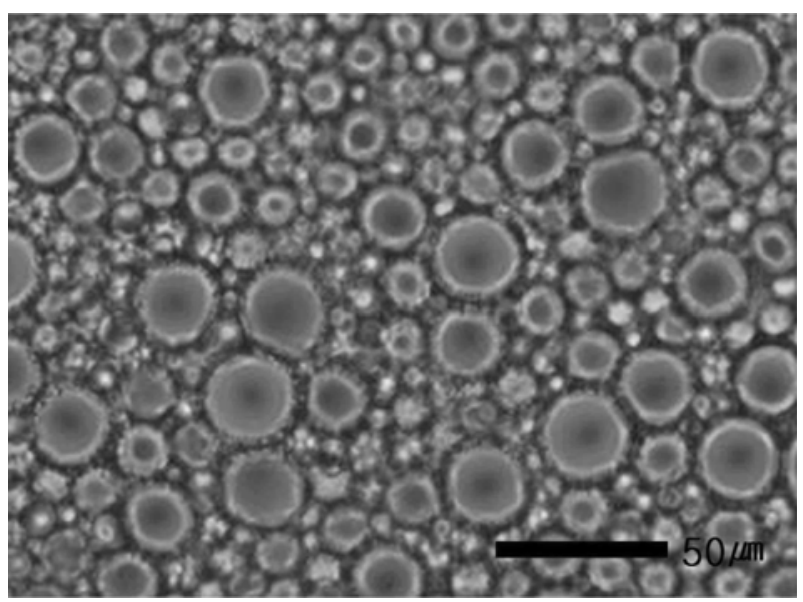

Fig. 8. Optical microscopy image of W/O emulsion $(\times 400)$ (TEOS : DC3225C : extracted dye=31.1 : $10.0: 58.9(w t \%))$.

Fig. 8 shows optical micrographs of the W/O type emulsion as an oil phase enclosing the water phase of the extracted dye. The emulsion state was checked in this analysis. Fig. 9 shows the particle size distribution according to the molar ratio of TEOS/ $\mathrm{H}_{2} \mathrm{O}$. The particle size increased with increasing TEOS/ $\mathrm{H}_{2} \mathrm{O}$ molar ratio.

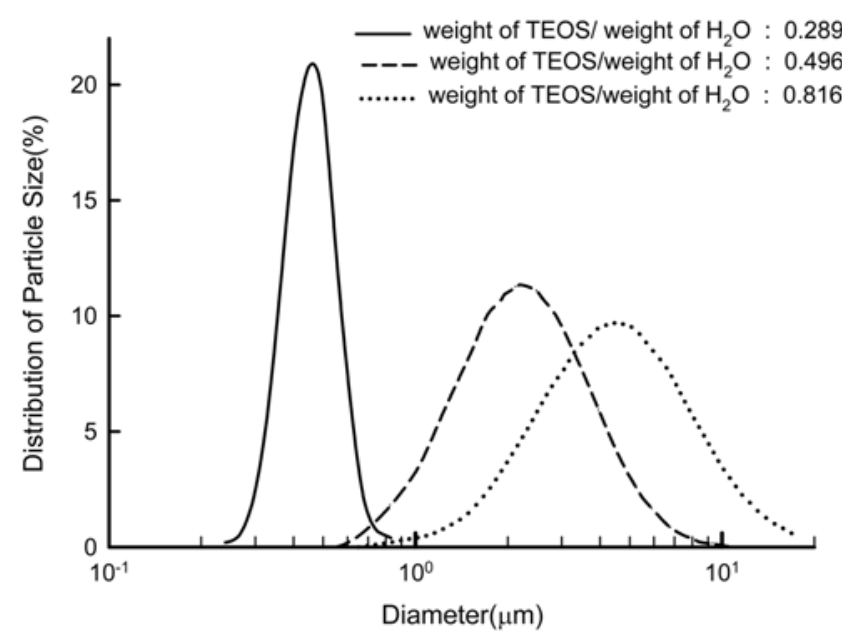

Fig. 9. Particle size distribution of W/O type emulsion according to TEOS/ $\mathrm{H}_{2} \mathrm{O}$ weight ratio (injection rate of extracted dye: $1.9 \mathrm{ml} / \mathrm{min}$; stirring rate: $750 \mathrm{rpm}$; DC3225C: $10 \mathrm{wt} \%$ ).

Table 1. Heavy metal analysis

\begin{tabular}{clc}
\hline \hline No & Heavy metal & Weight of heavy metal $\left(\mathrm{ppm}^{*}\right)$ \\
\hline 1 & $\mathrm{Ba}$ (barium) & 11.32 \\
2 & $\mathrm{Cr}$ (chromium) & 1.63 \\
3 & $\mathrm{~Pb}$ (lead) & No detection \\
4 & $\mathrm{Cd}$ (cadmium) & No detection \\
5 & $\mathrm{Sb}$ (antimony) & No detection \\
6 & $\mathrm{As}$ (arsenic) & No detection \\
7 & $\mathrm{Hg}$ (mercury) & No detection \\
8 & $\mathrm{Se}$ (selenium) & No detection \\
\hline
\end{tabular}

*ppm $=($ weight of heavy metal/weight of solution $) \times 10^{6}$.

The color paper made from emulsified extract was tested for the content of heavy metal and several properties of contained dye. The contents of the eight heavy metals that were restricted in the pigment and dye were measured by ICP. Table 1 shows the extremely small quantities of heavy metals extracted from sappan wood. The concentration of barium $(\mathrm{Ba})$ and chromium $(\mathrm{Cr})$ was $11.32 \mathrm{ppm}$ and $1.63 \mathrm{ppm}$, respectively, and no other heavy metals were detected. Table 2 shows the properties of the synthesized red ink from the extracted sappan wood. The test results satisfied the restriction conditions based on the Korea Food and Drug Administration Notice No. 2000-60, and the color fastness to sunlight based on the KS K 0700-1990 was $4^{\circ}$.

Table 2. The properties of the red ink made from the extracted sappan wood

\begin{tabular}{lccc}
\hline \hline Test item & Test result & Constraint $(\mathrm{mg} / \mathrm{L})$ & Test method \\
\hline Heavy metal $(\mathrm{Pb})$ & No detection & Below 1.0 & Korea Food and \\
Evaporation residue & 0.3 & Below 30 & Drug Administration \\
Formaldehyde & No detection & Below 4.0 & Notice No. 2000-60 \\
Fluorescent substance & No detection & No detection & KS K 0700-1990 \\
Arsenic & No detection & No detection & - \\
Color fastness & 4 degree & - & K
\end{tabular}




\section{CONCLUSION}

Colored components were extracted from sappan wood, and a W/O type emulsion of the extracted pigment was made by controlling the TEOS and DC $3225 \mathrm{C}$ concentrations. The highest absorbance was obtained by using the aluminum mordant. The optimum condition for the highest absorbance was obtained at an aluminum concentration of $2 \mathrm{wt} \%$. An emulsion of the extracted dye was obtained at TEOS concentrations ranging from 19.9 to $50.0 \mathrm{wt} \%$ when the DC3225C concentration was fixed to $10 \mathrm{wt} \%$. The applicability of the extracted natural dye to the nontoxic colored paper was tested.

\section{ACKNOWLEDGMENT}

The research was supported by Grants from the Small and Medium Business Administration. The authors would like to thank Micom Art Paper Co. for the assistance of acrylic resin and making colored paper.

\section{REFERENCES}

1. H. Zollinger, Color chemistry: syntheses, properties, and applications of organic dyes and pigments, Wiley-VCH, Zurich (1991).

2. G. A. F. Hendry and J. D. Houghton, Natural food colorants, Blackie,
New York (1996).

3. C. Y. Lee and W. J. Kim, Natural spice and food coloring, Hyanghaksa, Seoul (1985).

4. C. Fuke, J. Yamahara, T. Shimokawa, J.-E. Kinjo, T. Tomimatsu and T. Nohara, Phytochemistry, 24, 2403 (1985).

5. H.-X. Xu and S. F. Lee, Phytotherapy Research, 18, 647 (2004).

6. A. Ozaki, M. Kitano, N. furusawa, H. Yamaguchi, K. Kuroda and G. Endo, Food and Chemical Toxicology, 40, 1603 (2002).

7. I. Karapanagiotis and Y. Chryssoulakis, Annali Di Chimica, 96, 75 (2006).

8. Y. J. Chu, Journal of the Korean Society of Clothing and Textiles, 22, 971 (1998).

9. T. Nakashima, M. Shimizu and M. Kukizaki, Adavaced Drug Delivery Review, 45, 47 (2000).

10. K. J. Yong, I. H. Kim and S. W. Nam, Journal of the Korean Society of Dyes and Finishers, 11, 9 (1999).

11. S. R. Lee, Y. H. Lee, I. H. Kim and S. W. Nam, Journal of the Korean Society of Dyers and Finishers, 7, 374 (1995).

12. O. S. Cha and S. H. Kim, Journal of the Korean Society of Clothing and Textiles, 23, 788 (1999).

13. G. Sandberg, Indigo textiles, Lark Book, Asheville, North Carolina (1989).

14. E. K. Hwang, M. S. Kim, D. S. Lee and K. B. Kim, Journal of the Korean Fiber Society, 35, 490 (1998). 